\title{
PhyteByte: identification of foods containing compounds with specific pharmacological properties
}

\author{
Kenneth E. Westerman ${ }^{1,2}$, Sean Harrington ${ }^{3}$, Jose M. Ordovas ${ }^{1}$ and Laurence D. Parnell ${ }^{4^{*}}$ (D)
}

\author{
* Correspondence: laurence. \\ parnell@usda.gov \\ ${ }^{4}$ USDA Agricultural Research \\ Service, Nutrition and Genomics \\ Laboratory, JM-USDA Human \\ Nutrition Research Center on Aging \\ at Tufts University, Boston, MA, USA \\ Full list of author information is \\ available at the end of the article
}

\begin{abstract}
Background: Phytochemicals and other molecules in foods elicit positive health benefits, often by poorly established or unknown mechanisms. While there is a wealth of data on the biological and biophysical properties of drugs and therapeutic compounds, there is a notable lack of similar data for compounds commonly present in food. Computational methods for high-throughput identification of food compounds with specific biological effects, especially when accompanied by relevant food composition data, could enable more effective and more personalized dietary planning. We have created a machine learning-based tool (PhyteByte) to leverage existing pharmacological data to predict bioactivity across a comprehensive molecular database of foods and food compounds.
\end{abstract}

Results: PhyteByte uses a cheminformatic approach to structure-based activity prediction and applies it to uncover the putative bioactivity of food compounds. The tool takes an input protein target and develops a random forest classifier to predict the effect of an input molecule based on its molecular fingerprint, using structure and activity data available from the ChEMBL database. It then predicts the relevant bioactivity of a library of food compounds with known molecular structures from the FooDB database. The output is a list of food compounds with high confidence of eliciting relevant biological effects, along with their source foods and associated quantities in those foods, where available. Applying PhyteByte to the human PPARG gene, we identified irigenin, sesamin, fargesin, and delta-sanshool as putative agonists of PPARG, along with previously identified agonists of this important metabolic regulator.

Conclusions: PhyteByte identifies food-based compounds that are predicted to interact with specific protein targets. The identified relationships can be used to prioritize food compounds for experimental or epidemiological follow-up and can contribute to the rapid development of precision approaches to new nutraceuticals as well as personalized dietary planning.

Keywords: Bioactivity, Food, Molecule, Natural compound, Nutrition, Protein target 


\section{Background}

While a select set of essential nutrients for humans has been well characterized, there is an abundance of lesser-known compounds in the human diet, representing a type of exposure that has been referred to as the "dark matter" of the human exposome [1, 2]. These dietary bioactive compounds can have meaningful effects on human phenotypes, to the extent that some, such as lutein and several flavonoids, are under discussion for the establishment of dietary recommended intakes [3]. Despite the potentially important cumulative effects of these compounds, little is known about their bioactivity in the body due to the difficulty of experimentally assaying thousands of compounds for activity against thousands of potential gene products, combined with the complexities of absorption, microbial interactions, and metabolism [4]. Cheminformatic methods, including quantitative structure activity relationship (QSAR) models, can provide in silico approaches to prioritize compounds and foods in experimental and epidemiological settings when only the structure of a food compound is known. Pharmaceutical drugs can provide a critical set of anchors for such models, as their primary biological mechanisms of action are typically well characterized.

Computational approaches to generating hypotheses related to food and food compound bioactivity have been introduced $[5,6]$. However, existing methods have focused primarily on literature mining based on natural language processing, rather than optimizing for the output of food compound activities related to a given input gene or protein of interest. Methods described to date have used relatively basic QSAR methods, such as comparisons based on Tanimoto similarity scores, which may fail to capture important signals. Additionally, there can be significant utility in identifying the food(s) that contains a compound of interest both as a source material or in the formulation of a novel product. The growth of relevant databases containing pharmaceutical and food composition information continually offers opportunities to revisit and improve QSAR tools. The United States Department of Agriculture (USDA) has a long history of producing high-quality data for its food composition databases [7], and inclusion of established or potential health effects would be a useful extension of these data.

Here, we develop and demonstrate a machine learning-based approach, PhyteByte, that assigns putative bioactivity to food compounds based on a training set of pharmaceutical drugs. We show the efficacy of PhyteByte using the specific example of PPARG, the known target of the thiazolidinedione (TZD) drug class.

\section{Implementation}

In order to identify functional relationships between a food compound and a drug, along with its associated bioactivity data, we used data from two sources: ChEMBL and FooDB. ChEMBL is a manually curated database of almost 2 million $(1,879,206$ in version 25) bioactive molecules with drug-like properties $[8,9]$. These data were retrieved from ebi.ac.uk/chembl/ on 9/27/2019. FooDB (version 1.0) is a comprehensive resource on food constituents, chemistry and biology, with over 85,000 compounds in its repository [10]. These data were accessed from foodb.ca on 9/27/2019. As allele-specific binding data are not available in ChEMBL, PhyteByte currently does not have the means to incorporate genetic variants into its prediction. 
The PhyteByte computational pipeline is outlined in Fig. 1 (along with details related to a specific gene input; see Results \& Discussion). The processing of data through PhyteByte is initiated by selection of an input protein target query, from which drugs acting on that target (sourced from ChEMBL) are obtained to provide computational fingerprints of their molecular structure. The fingerprints are processed by a predictive model to yield likely bioactivity for food compounds (sourced from FooDB), which in turn are queried in FooDB to retrieve foods containing those compounds, with quantified amounts where available.

Specifically, a target specification (provided in the form of an HGNC gene symbol) serves as input for a query to ChEMBL that retrieves chemical structures for molecules

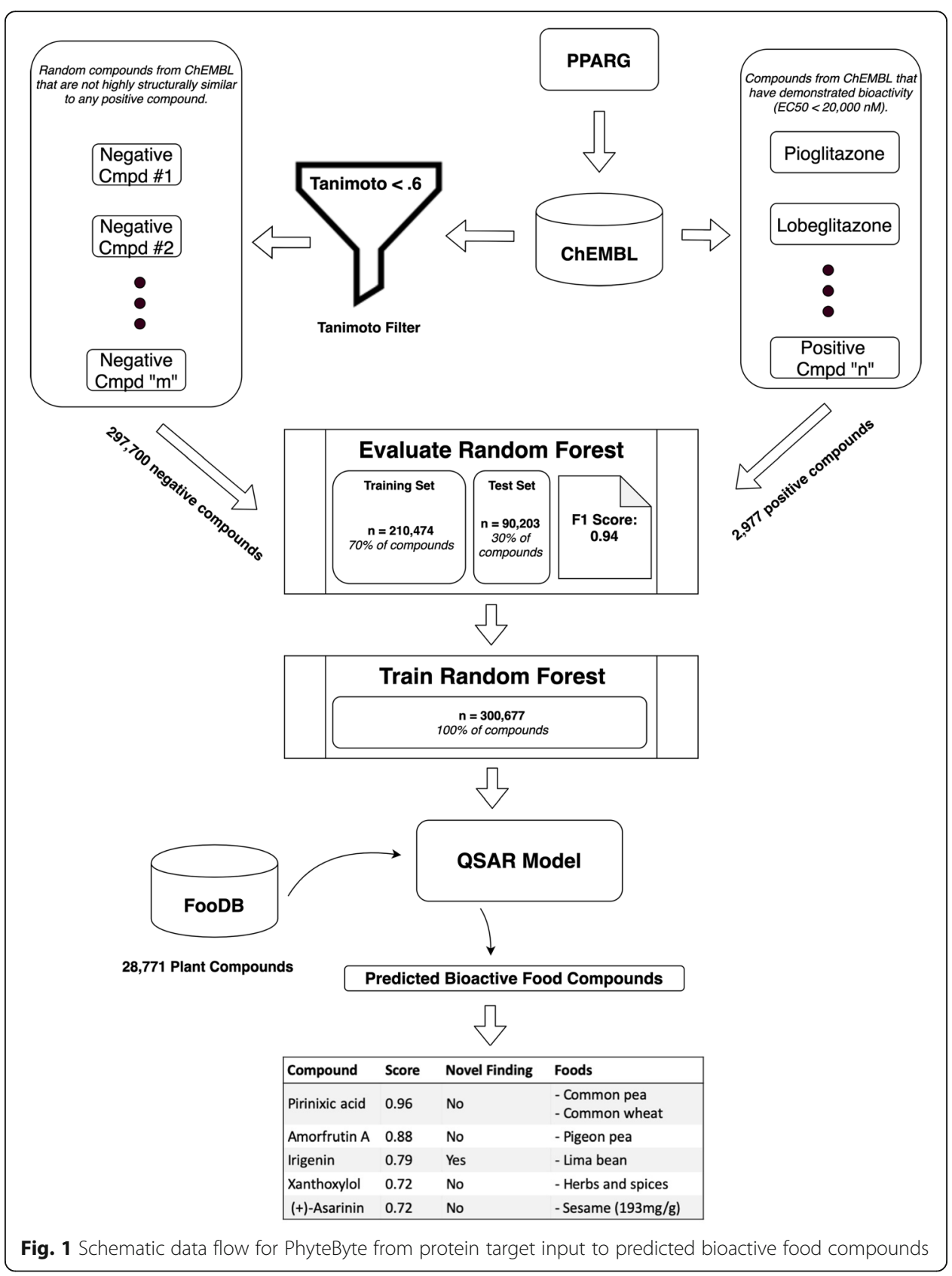


with evidence of relevant bioactivity for the protein encoded by that gene. Bioactivity is defined as an IC50 (inhibitory concentration: the concentration of the molecule required to inhibit the biochemical function of the target by 50\%) or EC50 (effective concentration; the concentration of the molecule required to induce $50 \%$ of the maximal response or effect on the target) of $<20,000 \mathrm{nM}$ based on the user-specified compound effect type (antagonist vs. agonist). Because ChEMBL does not contain explicit annotations as to the effect type, a heuristic is used in which the strength of antagonists and agonists are evaluated using IC50 and EC50 values, respectively. Compound structures are retrieved as simplified molecular-input line-entry system (SMILES) strings. SMILES strings are a dense, character-based representation of chemical compounds (for example, "COC1 $=\mathrm{CC}(=\mathrm{CC}(=\mathrm{C} 1 \mathrm{OC}) \mathrm{O}) \mathrm{C} 2=\mathrm{COC} 3=\mathrm{C}(\mathrm{C} 2=\mathrm{O}) \mathrm{C}(=\mathrm{C}(\mathrm{C}(=\mathrm{C} 3) \mathrm{O}) \mathrm{OC}) \mathrm{O}$ " for irigenin, a compound in Table 1). The SMILES strings are then converted into FP2 binary fingerprints using the Pybel Python package [11], which acts as a wrapper for the OpenBabel chemical file format interconversion tool. FP2 fingerprints are a binary compound representation (as a 1024-bit vector) formulated based on the occurrence of specific linear fragments up to 7 atoms in length. Further details on the SMILES and FP2 formats are available from the Open Babel publication [12] and online Wiki (https://openbabel.org). A set of negative examples, chosen to be 10 times the size of

Table 1 Top food compound results from PhyteByte for input of PPARG

\begin{tabular}{|c|c|c|c|c|c|c|}
\hline Compound & Synonyms & $\begin{array}{l}\text { CAS } \\
I D^{a}\end{array}$ & FooDB ID & Score $^{b}$ & $\begin{array}{l}\text { Novel } \\
\text { finding }\end{array}$ & Foods $^{c}$ \\
\hline Pirinixic acid & $\begin{array}{l}\text { 2-Methylthioribosyl-trans-zeatin; } \\
\text { WY-14,643; CXPTA }\end{array}$ & $\begin{array}{l}50892- \\
23-4\end{array}$ & FDB001402 & 0.96 & False & pea, wheat \\
\hline Amorfrutin A & $\begin{array}{l}\text { 3-Hydroxy-4-isopentenyl-5- } \\
\text { methoxybibenzyl-2-carboxylic acid }\end{array}$ & $\begin{array}{l}80489- \\
90-3\end{array}$ & FDB001743 & 0.88 & False & pigeon pea \\
\hline Irigenin & $\begin{array}{l}5,7,3^{\prime} \text {-Trihydroxy- } 6,4^{\prime}, 5^{\prime}- \\
\text { trimethoxyisoflavone }\end{array}$ & $\begin{array}{l}548- \\
76-5\end{array}$ & FDB008016 & 0.79 & True & $\begin{array}{l}\text { lima bean, iris } \\
\text { kemaonensis, } \\
\text { leopard lily }\end{array}$ \\
\hline Xanthoxylol & (-)-Piperitol & $\begin{array}{l}54983- \\
95-8\end{array}$ & FDB000580 & 0.72 & False & $\begin{array}{l}\text { herbs and } \\
\text { spices, } \\
\text { Asarum } \\
\text { sieboldii }\end{array}$ \\
\hline Sesamin & (+)-Asarinin; Fagarol & $\begin{array}{l}607- \\
80-7\end{array}$ & FDB012573 & 0.72 & False & $\begin{array}{l}\text { sesame, } \\
\text { flaxseed, fats } \\
\text { and oils }\end{array}$ \\
\hline $\begin{array}{l}\text { 2,3- } \\
\text { Dihydrobenzofuran }\end{array}$ & $\begin{array}{l}\text { 2,3-Dihydro-1-benzofuran; } \\
\text { Coumaran; Dihydrocoumarone }\end{array}$ & $\begin{array}{l}496- \\
16-2\end{array}$ & FDB007352 & 0.72 & True & fenugreek \\
\hline$(+)$-Fargesin & $\begin{array}{l}\text { (+)-Spinescin; 2-(3',4'- } \\
\text { Dimethoxyphenyl)-6-(3",4"-- } \\
\text { methylenedioxyphenyl)-3,7- } \\
\text { dioxabicyclo(3,3,0)octane; } \\
\text { Methylpluviatilol; Planinin }\end{array}$ & $\begin{array}{l}68296- \\
27-5\end{array}$ & FDB017481 & 0.69 & True & $\begin{array}{l}\text { tea, herbs } \\
\text { and spices }\end{array}$ \\
\hline delta-Sanshool & $\begin{array}{l}\text { N-Isobutyl-2,4,8,10,12- } \\
\text { tetradecapentaenamide; g-Sanshool }\end{array}$ & $\begin{array}{l}78886- \\
65-4\end{array}$ & FDB003203 & 0.65 & True & $\begin{array}{l}\text { herbs and } \\
\text { spices } \\
\text { (general) }\end{array}$ \\
\hline Sanshodiol & $\begin{array}{l}\text { (5-Chloro-2-hydroxyphenyl) acetic } \\
\text { acid }\end{array}$ & $\begin{array}{l}54854- \\
91-0\end{array}$ & FDB002461 & 0.65 & True & $\begin{array}{l}\text { herbs and } \\
\text { spices }\end{array}$ \\
\hline Samin & & NA & FDB018392 & 0.61 & True & fats and oils \\
\hline
\end{tabular}


the positive set, is also retrieved at random from the full set of ChEMBL molecules. The negative examples are converted to FP2 fingerprints after filtering such that no negative compound has a Tanimoto similarity score $>0.6$ with any molecule in the positive set. The Tanimoto coefficient is defined as an association coefficient (in comparison to a distance coefficient) that measures similarity, here as chemical similarity based on SMILES representation of the molecule [13]; formulae for the Tanimoto coefficient are presented elsewhere [14]. No explicit upper limit for molecular mass of the bioactive molecules is set, but we note that the vast majority $(>98 \%)$ of molecules in ChEMBL are categorized as small molecules.

Next, a random forest model is trained (using the sklearn Python package) to classify compounds as to their bioactivity against the protein of interest. Inputs consist of the binary fingerprints (a binary feature vector of length 1024) and class labels (positive if evidence of bioactivity for the target exists in ChEMBL, or negative if not). The random forest classifier is an ensemble learning method that trains a set of independent decision trees to discriminate between positive and negative examples. Given a new compound (in this case, a food compound), binary predictions from each individual decision tree are averaged to output a probability of bioactivity. Models in PhyteByte use 100 component trees, with all additional parameters following sklearn defaults. The training and testing dataset split is created by assigning a random $30 \%$ of compounds to the testing dataset (including a consistent random seed for reproducibility), with the remaining $70 \%$ assigned to the training dataset. We note that after evaluation, the final model used to process food compounds is trained on the full dataset. An initial indication of model performance is evaluated in a 30\% held-out testing set using the F1 score, or the harmonic mean of precision and recall. This metric is calculated as $F_{1}=2 *$ $\frac{\text { precision*recall }}{\text { precision }+ \text { recall }}$ where precision is the fraction of predicted bioactive compounds that have evidence for bioactivity in ChEMBL, and recall is the fraction of compounds with evidence for bioactivity in ChEMBL that are predicted to be bioactive. True positive (TP) is defined as bioactivity in ChEMBL and predicted to be bioactive; false positive (FP) is defined as no bioactivity in ChEMBL but predicted to bioactive; false negative (FN) is defined as bioactivity in ChEMBL but not predicted to be bioactive. Thus, precision = $\mathrm{TP} /(\mathrm{TP}+\mathrm{FP})$, recall $=\mathrm{TP} /(\mathrm{TP}+\mathrm{FN})$, and F1 is calculated as above.

Using this trained model, the full set of food compounds available from FooDB are then characterized as to their probability of bioactivity with respect to the input protein. The list of probable dietary bioactive compounds is presented as output, along with their concentrations in foods as available in FooDB and an indication of whether the relationship is novel (i.e. does the compound lack existing evidence of bioactivity for the input protein in ChEMBL?). PhyteByte source code and installation instructions are available at https://github.com/seanharr11/phytebyte, accompanied by the readme text file with its detailed information of code usage in Additional file 1.

\section{Results \& discussion}

We have demonstrated the functionality and output of PhyteByte using the input gene PPARG (CHEMBL235), whose protein product is the target of the thiazolidinedione (TZD) drug class. TZDs are widely prescribed to treat type 2 diabetes, and additionally may have broader cardiometabolic benefits [15]. However, TZDs also have documented 
side effects and FDA-issued alerts of adverse effects [16], suggesting a potential benefit of identifying alternative or complementary food-based bioactives. Details of the PhyteByte pipeline as realized for PPARG agonists are presented in Fig. 1. 2977 positive compounds were retrieved from ChEMBL, along with 297,700 negative compounds. The trained model exhibited an F1 score (harmonic mean of precision and recall) of 0.94 in a $30 \%$ held-out set, indicating a reasonably strong discriminative capacity within the set of molecules in ChEMBL. This score may be biased upwards due to limitations in the set of pharmaceutical compounds explored to date, but nonetheless indicates an ability to classify potential food compounds effectively.

When used to score compounds from FooDB, the model identified a series of molecules with potential agonist bioactivity for PPARG. Table 1 lists the 10 molecules with a predicted bioactivity confidence of greater than 0.60 that also had associated foods in FooDB; tabulated results include the identified food compound, common synonyms, CAS and FooDB identifiers, PhyteByte output score, whether the compound-PPARG interaction is a novel finding, and foods reported to contain that compound. Molecules such as pirinixic acid (or WY-14643) and xanthoxylol have been shown to activate PPARG [17-19], albeit the latter only as an activator of PPARG transcription [20]. Other molecules have little to no existing evidence in the scientific literature of acting as PPARG agonists. These include irigenin (an O-methylated flavone found in lima bean), sesamin (a lignan found in sesame and flaxseed), fargesin (a lignan from tea, herbs and spices), delta-sanshool (an n-acyl amine from herbs and spices), and the lignan sanshodiol (from herbs and spices). Such molecules could be prioritized for detailed experimental validation. Complete output of PhyteByte for PPARG as input and resulting identified compounds scoring above 0.50 is presented in Additional file 2.

Tools such as PhyteByte consider only small molecules and are limited by the content of the input databases. Importantly, these resources are expected to become increasingly comprehensive, especially for food compounds. For example, efforts are underway by the USDA to expand their food composition databases [7], and recent investigations have identified additional compounds produced during food processing [21] and by human microbiota [22], which may promote certain health effects. While QSAR models are susceptible to false positives due to activity cliffs (key discontinuities in the structure-activity landscape), outputs from PhyteByte are intended to be only putative structure-activity relationships to be explored further through complementary computational and laboratory methods [23]. Experimental and/or epidemiological assessment eventually will be required to validate at least some subset of the algorithmic predictions before this tool could be used in clinical settings or for dietary recommendations.

In future versions of the software, we anticipate more flexibility in both the inputs and databases. For example, inputs may include phenotypes (to be linked to a set of target gene products), and user-defined food compound datasets following a predefined schema may be used to complement FooDB. Additionally, as more follow-up testing of food compound-target interactions is performed, those results can be used as a complementary source of interactions for PhyteByte and form the basis for a catalog of all such interactions for a single food. Complementary data streams, such as those based on text mining [5], pharmacology networks [24] or drug interaction data (to identify potential similar food compound interaction effects), could provide additional support for food compound-phenotype links. Future work also should include more 
fine-grained annotations of positive training molecules (based on type of effect on the target, strength, and mechanism of action) as well as alternative QSAR modeling approaches [25].

\section{Conclusions}

PhyteByte is a machine learning-based tool for discovery of interactions between food compounds and specific proteins or phenotypes. The software enables prioritization of these compounds for future research and hypothesis generation for condition-specific dietary interventions. Applied to the PPARG gene, this tool recovered known ligands and generated the basis for new hypotheses useful for cell-based assays or epidemiological inquiries. Our work provides additional proof-of-concept for the emerging field of "computational nutrition" based on food compounds, building on previous research that applied cheminformatic approaches to assign putative biological function to molecules of interest.

\section{Availability and requirements}

Project name: Phytebyte.

Project home page: https://github.com/seanharr11/phytebyte

Operating system(s): Unix-based (MacOS, Linux).

Programming language: Python.

Other requirements: Python 3.6 or higher.

License: AGPLv3.

Any restrictions to use by non-academics: License needed.

\section{Supplementary information}

Supplementary information accompanies this paper at https://doi.org/10.1186/s12859-020-03582-7.

Additional file 1.

Additional file 2.

\section{Abbreviations}

EC50: Effective concentration; IC50: Inhibitory concentration; PPARG: Peroxisome proliferator activated receptor gamma; QSAR: Quantitative structure activity relationship; SMILES: Simplified molecular-input line-entry system; TZD: Thiazolidinedione; USDA: United States Department of Agriculture

\section{Acknowledgements}

Mention of trade names or commercial products in this publication is solely for the purpose of providing specific information and does not imply recommendation or endorsement by the U.S. Department of Agriculture. The USDA is an equal opportunity provider and employer.

\section{Authors' contributions}

All authors had essential and integrated roles in this project. All authors conceived of, and KEW, SH and LDP designed, wrote and tested PhyteByte, and/or analyzed results. KEW, SH and LDP wrote, and all authors reviewed, revised and approved the submitted manuscript. JMO contributed to both quality metrics and intellectual guidance during conception of the software, analyzed results, and provided financial support to KEW and LDP.

\section{Funding}

This work was funded in part by United States Department of Agriculture project number 8050-51000-107-00D (awarded to $\mathrm{JMO}$ ), and this entity had no part in the design of this software, collection, analysis and interpretation of data, nor in composing the manuscript. 


\section{Consent for publication}

Not applicable.

\section{Competing interests}

$\mathrm{SH}$ is a founder and employee of Notemeal, Inc., a company building a software platform for performance dietitians to manage athlete nutrition. This entity currently has no relation to or use of the findings described here. All other authors declare that they have no competing interests.

\section{Author details}

${ }^{1}$ Nutrition and Genomics Laboratory, JM-USDA Human Nutrition Research Center on Aging at Tufts University, Boston, MA, USA. ${ }^{2}$ Clinical and Translational Epidemiology Unit, Massachusetts General Hospital, Boston, MA, USA. ${ }^{3}$ Notemeal, Inc., Boston, MA, USA. ${ }^{4}$ USDA Agricultural Research Service, Nutrition and Genomics Laboratory, JM-USDA Human Nutrition Research Center on Aging at Tufts University, Boston, MA, USA.

Received: 7 January 2020 Accepted: 3 June 2020

Published online: 10 June 2020

\section{References}

1. Uppal K, Walker DI, Liu K, Li S, Go YM, Jones DP. Computational metabolomics: a framework for the million metabolome. Chem Res Toxicol. 2016;29(12):1956-75.

2. Barabási AL, Menichetti G, Loscalzo J. The unmapped chemical complexity of our diet. Nat Food. 2020;1:33-7.

3. Wallace TC, Blumberg JB, Johnson EJ, Shao A. Dietary bioactives: establishing a scientific framework for recommended intakes. Adv Nutr. 2015;6(1):1-4.

4. Rein MJ, Renouf M, Cruz-Hernandez C, Actis-Goretta L, Thakkar SK, da Silva Pinto M. Bioavailability of bioactive food compounds: a challenging journey to bioefficacy. Br J Clin Pharmacol. 2013;75(3):588-602.

5. Jensen K, Panagiotou G, Kouskoumvekaki I. NutriChem: a systems chemical biology resource to explore the medicinal value of plant-based foods. Nucleic Acids Res. 2015;43(Database issue):D940-5.

6. Ni Y, Jensen K, Kouskoumvekaki I, Panagiotou G. NutriChem 2.0: Exploring the effect of plant-based foods on human health and drug efficacy. Database (Oxford). 2017;2017:1-6.

7. Haytowitz DB, Pehrsson PR. USDA's National Food and nutrient analysis program (NFNAP) produces high-quality data for USDA food composition databases: two decades of collaboration. Food Chem. 2018;238:134-8.

8. Gaulton A, Hersey A, Nowotka M, Bento AP, Chambers J, et al. The ChEMBL database in 2017. Nucleic Acids Res. 2017; 45(D1):D945-54

9. ChEMBL. http://www.ebi.ac.uk/chembl/. Accessed 27 Sep 2019

10. FooDB. A resource on food constituents, chemistry and biology. foodb.ca. Accessed 27 Sep 2019

11. O'Boyle NM, Morley C, Hutchison GR. Pybel: a Python wrapper for the OpenBabel cheminformatics toolkit. Chem Cent J. 2008;2:5.

12. O'Boyle NM, Banck M, James CA, Morley C, Vandermeersch T, Hutchison GR. Open babel: an open chemical toolbox. Aust J Chem. 2011;3:33.

13. Melville JL, Riley JF, Hirst JD. Similarity by compression. J Chem Inf Model. 2007;47(1):25-33.

14. Willett P, Barnard JM, Downs GM. Chemical similarity searching. J Chem Inf Comput Sci. 1998;38(6):983-96.

15. Liu J, Wang LN. Peroxisome proliferator-activated receptor gamma agonists for preventing recurrent stroke and other vascular events in patients with stroke or transient ischaemic attack. Cochrane Database Syst Rev. 2015;29(10):CD010693.

16. Hong F, Xu P, Zhai Y. The opportunities and challenges of peroxisome proliferator-activated receptors ligands in clinical drug discovery and development. Int J Mol Sci. 2018;19(8):2189.

17. Zhou YC, Waxman DJ. Activation of peroxisome proliferator-activated receptors by chlorinated hydrocarbons and endogenous steroids. Environ Health Perspect. 1998;106(Suppl 4):983-8.

18. Knowles HJ, te Poele RH, Workman P, Harris AL. Niacin induces PPARgamma expression and transcriptional activation in macrophages via HM74 and HM74a-mediated induction of prostaglandin synthesis pathways. Biochem Pharmacol. 2006;71(5):646-56.

19. Temkin AM, Bowers RR, Magaletta ME, Holshouser S, Maggi A, et al. Effects of crude oil/dispersant mixture and dispersant components on PPARy activity in vitro and in vivo: identification of dioctyl sodium sulfosuccinate (DOSS; CAS \#577-11-7) as a probable obesogen. Environ Health Perspect. 2016;124(1):112-9.

20. Quang TH, Ngan NT, Minh CV, Kiem PV, Tai BH, et al. Anti-inflammatory and PPAR transactivational effects of secondary metabolites from the roots of Asarum sieboldii. Bioorg Med Chem Lett. 2012;22(7):2527-33.

21. Gauglitz JM, Aceves CM, Aksenov AA, Aleti G, Almaliti J, et al. Untargeted mass spectrometry-based metabolomics approach unveils molecular changes in raw and processed foods and beverages. Food Chem. 2020;302:125290.

22. Rowland I, Gibson G, Heinken A, Scott K, Swann J, et al. Gut microbiota functions: metabolism of nutrients and other food components. Eur J Nutr. 2018:57(1):1-24.

23. Stumpfe D, Hu Y, Dimova D, Bajorath J. Recent progress in understanding activity cliffs and their utility in medicinal chemistry. J Med Chem. 2014;57(1):18-28.

24. Zhang B, Wang X, Li S. An integrative platform of TCM network pharmacology and its application on a herbal formula, Qing-Luo-Yin. Evid Based Complement Alternat Med. 2013;2013:456747.

25. Neves BJ, Braga RC, Melo-Filho CC, Moreira-Filho JT, Muratov EN, Andrade CH. QSAR-based virtual screening: advances and applications in drug discovery. Front Pharmacol. 2018;9:1275.

\section{Publisher's Note}

Springer Nature remains neutral with regard to jurisdictional claims in published maps and institutional affiliations. 